\title{
Estratégias de Aprendizagem e Desempenho Acadêmico: Evidências de Validade
}

\author{
Katya Luciane de Oliveira ${ }^{1}$ \\ Universidade Estadual de Londrina \\ Evely Boruchovitch \\ Unicamp \\ Acácia Aparecida Angeli dos Santos \\ Universidade São Francisco
}

\begin{abstract}
RESUMO - O objetivo deste estudo foi verificar a validade fatorial de uma escala de estratégias de aprendizagem, bem como explorar sua validade concorrente em relação ao desempenho acadêmico de estudantes. Participaram 815 crianças do Ensino Fundamental de escolas públicas e privadas dos Estados de São Paulo e Minas Gerais. A Escala de Estratégias de Aprendizagem foi aplicada coletivamente. Para a operacionalização dos objetivos, recorreu-se à análise fatorial exploratória. Os alphas de Cronbach do instrumento e das três subescalas revelaram índices aceitáveis de consistência interna. A análise de variância apontou diferenças estatisticamente significativas entre o desempenho acadêmico e a pontuação na escala. Os dados foram discutidos em termos de suas possíveis implicações para a área de avaliação psicoeducacional.
\end{abstract}

Palavras-chave: aprendizagem; estudo; avaliação psicoeducacional; estratégias de aprendizagem.

\section{Learning Strategies and Academic Performance: Validity Evidences}

\begin{abstract}
The purpose of the present study was to verify the factorial validity of a learning strategy scale as well as to explore the concurrent validity of the instrument in regard to students' academic achievement. The sample was composed of 815 basic education children from both public and private schools of São Paulo and Minas Gerais. The Learning Strategy Scale was collectively applied. Exploratory factorial analyses were conducted to achieve the purposes of the study. The alphas of Cronbach of the instrument and of its three subscales showed good reliability. Variance analyses showed significant differences between school achievement and punctuation in the scale. The data were discussed in terms of their possible implications for the psycho-educational evaluation area.
\end{abstract}

Keywords: learning; studying; psycho-educational evaluation; learning strategies.

Há uma concepção na psicologia, a qual ganhou forma na década de 1970, que, ao considerar o funcionamento da mente humana como análogo ao funcionamento de uma máquina, expandiu as ideias sobre o processamento da informação a fim de explicar a cognição humana. Dessa forma, a teoria do processamento da informação tem sido constantemente utilizada por teóricos da psicologia cognitiva, para quem a mente humana funcionaria como uma estrutura básica que processa e armazena todas as informações e impressões recebidas do meio. Os estudiosos do processamento humano da informação defendem que existe uma interação entre fatores contextuais e internos na aprendizagem (Bzuneck, 2004).

Autores como Dembo (1994), Nori e Giusbert (2006) e Sternberg (1996/2000) consideram que a aprendizagem se concretiza quando uma informação nova é armazenada na memória a longo prazo. $\mathrm{O}$ ambiente é o agente que promove a estimulação do raciocínio para que uma nova informação comece a ser processada.

1 Endereço para correspondência: Universidade Estadual de Londrina, Depto de Psicologia e Psicanálise. Rodovia Celso Garcia Cid, Km 380. Caixa Postal 6001. Londrina, PR. CEP 86051-980.E-mail: katya@uel. bre katya_lincoln@ig.com.br.
Nesse contexto, as estratégias de aprendizagem se inserem no processamento da informação como recursos valiosos que o estudante pode dispor no momento do estudo, visando maximizar a recuperação e a imediata utilização da informação. Nessa direção, Almeida (2002) menciona que o processo de aprendizagem do aluno deve ser ativo. A aprendizagem significativa deve ocorrer por meio de métodos que promovam a iniciativa e a responsabilidade do aluno no seu próprio aprendizado. As estratégias de aprendizagem funcionam como reforçadoras da aprendizagem visto que instrumentalizam o aluno a diversificar as formas de estudo, promovendo atitudes de autoavaliação e melhora do desempenho escolar.

Boruchovitch (1999) e Boruchovitch e Santos (2004a) constataram que as estratégias de aprendizagem podem, entre outras possíveis taxionomias, ser divididas em dois grandes grupos: estratégias cognitivas e metacognitivas. As estratégias cognitivas auxiliam os estudantes a operar diretamente com a informação. O uso desse tipo de estratégia reflete na forma do estudante organizar, armazenar e elaborar as informações (Dembo, 1994; Jamieson, 1995). Já as estratégias metacognitivas podem ser consideradas como recursos que o aluno utiliza para planejar, monito- 
rar e regular o seu próprio pensamento. Dembo (1994) e Veenman, Wilhelm e Beishuizer (2004) esclarecem que as estratégias metacognitivas são as mais complexas visto que têm uma função de administração do processo e envolvem o autoconhecimento da pessoa sobre o processo de aprender. As estratégias de planejamento, monitoramento e regulação da aprendizagem são consideradas metacognitivas (Boruchovitch \& Santos, 2006).

A metacognição é usada pelo aluno para que ele reflita sobre sua própria aprendizagem, buscando novas soluções para aprender de modo a conseguir superar obstáculos. O uso frequente das estratégias metacognitivas favorece o sucesso escolar e uma melhor apropriação do conhecimento (Boruchovitch \& cols., 2006; Darsie, 1996; Oliveira, Boruchovitch \& Santos, 2006; Zimmerman \& Martinez-Pons, 1986). As habilidades metacognitivas, segundo Veenman e cols. (2004), são preditoras da aprendizagem e se associam à inteligência, por apresentarem elementos analíticos do contexto de aprendizagem. Mais precisamente, a metacognição envolve, simultaneamente, o estabelecimento de objetivos de estudo (planejamento), o conhecimento sobre a própria compreensão (monitoramento) e o conhecimento de como compreender (regulação). Decorre daí a complexidade e a dificuldade de mensuração desse construto. Assim, pode-se concluir que a metacognição é um construto bastante complexo.

Nessa perspectiva, o aprender a aprender se centra na construção de um tipo de conhecimento específico, denominado como estratégico (Valdés, 2003). Ser estratégico é muito mais do que utilizar técnicas e métodos para aprender. $\mathrm{O}$ estudante que emprega estratégias é um construtor à medida que tem um papel ativo e de mediação social do seu conhecimento.

Para Mckeachie, Pintrich e Lin (1985) e Reay (2006), a escola é a responsável pelo incentivo ao uso de estratégias de aprendizagem adequadas. Desse modo, conhecer estratégias que facilitam o armazenamento e a utilização dos novos conhecimentos é um desafio que pode se reverter, por um lado, na remediação das dificuldades de estudo e aprendizagem. Por outro lado, implicam a melhora do desempenho acadêmico nas diferentes séries formais de ensino. Autores estrangeiros e brasileiros defendem que o estudante que sabe utilizar adequadamente as estratégias de aprendizagem poderá apresentar um melhor desempenho acadêmico (Bzuneck, 2001; Tapia \& Garcia-Celay, 1996).

Costa e Boruchovitch (2000) realizaram uma revisão da literatura e fizeram uma análise crítica das pesquisas nacionais e internacionais sobre emprego e uso das estratégias de aprendizagem. Nos materiais consultados, evidências provenientes de investigações, em diferentes séries do ensino formal, convergem no que concerne à relação positiva entre o uso adequado das estratégias de aprendizagem e um melhor desempenho acadêmico.

No que se refere ao desempenho acadêmico, de uma forma geral, observa-se que o rendimento dos alunos tem um escore final que pode ser representado de diversas maneiras. Assim sendo, podem incluir números em um escala de 0 a 10 ou 0 a 100, letras tais como A, B, C, D e E, bem como classificações como Excelente, Ótimo, Bom, Regular e Péssimo. O desempenho final também pode ser emitido por meio de escalas que se restringem a 'aprovado' e 'reprova- do' ou 'satisfatório e 'insatisfatório'. O desempenho obtido por meio da avaliação da aprendizagem pode ser positivo quando possibilita ao aluno uma maior consciência de suas dificuldades (Buriasco, 2000).

Autores como Esteban (2000), Kasai (2000) e Martins (1999) discutem que o desempenho acadêmico deveria servir para que o professor pudesse de fato dar um novo sentido à aprendizagem de seu aluno, de modo que tal desempenho pudesse servir de base para que novas diretrizes de estudo fossem adotadas. Nessa direção, pode-se aventar que não somente o desempenho acadêmico poderia servir como critério para um melhor planejamento de estudo como também as estratégias de aprendizagem utilizadas no momento do estudo poderiam ser levantadas a fim de se poder orientar o aluno quanto ao seu emprego e diversificação no momento do estudo.

Alguns estudos foram realizados com a finalidade de se investigar as possíveis relações entre o uso das estratégias de aprendizagem e o bom desempenho acadêmico dos estudantes (Loranger, 1994; Moura, 1992; Onatsu-Arvilommi, Nurmi \& Aunola, 2002; Purdie \& Harttie, 1996). Esses estudos eram do tipo descritivo com delineamento de levantamento e correlacional, e todos evidenciaram que os estudantes que faziam uso de um repertório diversificado de estratégias de aprendizagem eram aqueles que também apresentavam um bom desempenho acadêmico.

Sob essa perspectiva, e considerando a importância das estratégias de aprendizagem para o bom desempenho acadêmico, aponta-se que, no Brasil, poucos são os instrumentos válidos e padronizados destinados a mensurar as estratégias de aprendizagem de estudantes brasileiros. Importante ressaltar, por fim, que estudiosos da área (Noronha, 1999; Noronha \& Alchieri, 2001; Sisto, Sbardelini \& Primi, 2001) têm denunciado insistentemente a carência de estudos que permitam evidenciar as propriedades psicométricas dos diferentes instrumentos de medida.

Portanto, tendo em vista a importância do uso de estratégias adequadas para a aprendizagem e a carência de instrumentos válidos para mensurá-las em nossa realidade, esta pesquisa se propõe a identificar as evidências de validade fatorial da Escala de Estratégias de Aprendizagem, versão 37 itens, de Boruchovitch e Santos (2004b). Também se propõe a explorar outra evidência de validade concorrente, baseada no desempenho acadêmico dos estudantes.

\section{Método}

\section{Participantes}

Participaram do presente estudo 815 estudantes das $2^{\mathrm{a}}, 3^{\mathrm{a}}$, $4^{\mathrm{a}}, 5^{\mathrm{a}}, 6^{\mathrm{a}}, 7^{\mathrm{a}}$ e $8^{\mathrm{a}}$ séries do Ensino Fundamental, com média de idade de 11 anos e 8 meses $(D P=1,9)$. A idade mínima foi de 7 anos e a máxima, 16. O gênero masculino representou $48,5 \%(n=395)$ da amostra e o feminino, $51,3 \%(n=418)$, sendo que duas pessoas $(0,2 \%)$ não responderam essa questão. Os estudantes eram provenientes de escolas públicas $(83 \%$; $n=676)$ e privadas $(17 \% ; n=96)$ dos estados de Minas Gerais $(34,8 \% ; n=284)$ e São Paulo $(65,2 \% ; n=531)$. 


\section{Instrumentos}

Ficha de identificação dos estudantes. Trata-se de um conjunto de questões sobre nome, sexo, idade, escola e série. As questões da ficha foram incluídas no próprio cabeçalho da escala de estratégias, descrita a seguir.

Escala de Estratégias de Aprendizagem (Boruchovitch \& Santos, 2004b). A construção dessa escala se baseou em instrumentos internacionais e em estudos anteriores (Boruchovitch \& Santos, 2004a; Boruchovitch \& cols. 2006; Oliveira \& cols., 2006). A escala de Boruchovitch e Santos (2004b) é composta por 37 itens, dos quais 20 itens são provenientes da escala anterior (Boruchovitch \& Santos, 2004a), cuja análise fatorial por componentes principais e rotação varimax mostrou a existência de três fatores, e por mais 17 novos itens, que foram submetidos à análise de juízes especialistas em estratégias de aprendizagem para a aferição de sua validade de conteúdo. A concordância entre eles ficou em $80 \%$.

Os assuntos abordados no instrumento são relativos às estratégias utilizadas no estudo e na aprendizagem. As questões abarcam as estratégias cognitivas e, como exemplo, cita-se a seguinte questão: 'Você costuma grifar as partes importantes do texto para aprender melhor?'. Referem-se também às estratégias metacognitivas e, como ilustração, cita-se: 'Quando você estuda, percebe se não está conseguindo aprender?'. As questões também abordam a ausência de estratégias de aprendizagem no estudo, como é o caso desta questão: 'Você costuma se distrair ou pensar em outra coisa quando está lendo ou fazendo o dever de casa?'. As alternativas de respostas são dispostas em escala Likert de três pontos: sempre, às vezes e nunca. A opção 'sempre' vale 3 pontos, a opção 'às vezes', 2 pontos e a opção 'nunca', 1 ponto. Alguns itens (3, 9, 10, 15, $19,23,26,28,29,30,31,33$ e 36) tiveram essa pontuação invertida, devido ao teor de sua redação.

\section{Procedimento}

Cabe mencionar que esta pesquisa atendeu a todos os dispositivos da Resolução 196/96 do Conselho Nacional de Saúde, que trata de pesquisas com seres humanos. As aplicações da Escala de Estratégias de Aprendizagem foram realizadas coletivamente nas escolas públicas e privadas de São Paulo e Minas Gerais para os pais que consentiram na participação do filho por meio da assinatura do Termo de Consentimento Livre e Esclarecido. Os pais também autorizaram que o pesquisador levantasse, junto às secretarias de cada instituição de ensino, as médias semestrais das disciplinas de Português e Matemática.

\section{Análise de dados}

No que concerne aos procedimentos de análise de dados, cabe mencionar, sucintamente, que para uma melhor operacionalização dos objetivos, os dados foram submetidos às provas de estatística descritiva (médias e desvios-padrão) e inferencial (Análise Fatorial Exploratória e Análise de Variância-ANOVA).

\section{Resultados}

Inicialmente, realizou-se uma análise estatística descritiva da Escala de Estratégias de Aprendizagem, de Boruchovitch e Santos (2004b). Os dados revelaram que a média de pontos na escala foi de 79,3 $(D P=10,0)$, sendo a pontuação mínima igual a 15 pontos e a máxima igual a 106 pontos.

Em seguida, buscou-se alcançar o primeiro objetivo específico deste estudo que se referiu ao exame da validade fatorial da referida escala. Para verificar se haveria a possibilidade de aplicar o método da análise fatorial exploratória, realizou-se o Teste de Esfericidade de Bartlett, que indicou uma correção entre os itens $\left(\chi^{2}[666 ; N=815]=4754,541\right.$; $p<0,001)$. A medida de adequação da amostra foi averiguada pelo índice de Kaiser-Meyer-Olkin (KMO) que foi de 0,869 .

A análise fatorial por componentes principais e rotação varimax indicou uma estrutura de três fatores para a Escala de Estratégias de Aprendizagem, com eigenvalues acima de 1,0 e com $29,4 \%$ da variância total explicada. O agrupamento dos fatores ficou assim distribuído: Fator 1- Ausência de Estratégias de Aprendizagem (itens 3, 9, 10, 15, 19, 23, 26, 28, 29, 30, 31, 33 e 36); Fator 2 - Estratégias Cognitivas (itens 1, 2, 4, 5, 6, 7, 11, 12, 13, 14, 16, 18, 20, 21, 24, 25 e 34); Fator 3 - Estratégias Metacognitivas (itens 8, 17, 22, $27,32,35$ e 37). A Tabela 1 mostra a distribuição dos itens por fator e suas respectivas cargas fatoriais.

O alpha de Cronbach da escala toda foi de 0,83 . Os alphas das subescalas foram: 0,79 para Ausência de Estratégias de Aprendizagem, 0,80 para Estratégias Cognitivas e 0,62 para Estratégias Metacognitivas. Contudo, alguns itens (6, 7, 14, 16,24 e 34) deverão ser mais bem explorados, visto que carregaram em fator não previsto teoricamente. No caso específico, eram itens aparentemente de Estratégias Metacognitivas que se aglutinaram a outros, presumidamente referentes às Estratégias Cognitivas. A Tabela 2 apresenta os itens que, possivelmente, deverão ser excluídos, discriminado o tipo de estratégias esperadas na rotação que não se confirmaram com a análise.

No segundo objetivo específico, recorreu-se à Análise de Variância - ANOVA para se identificar possíveis diferenças entre o desempenho acadêmico dos estudantes $(51,4 \% ; n=434)$ nas disciplinas de português e matemática e a pontuação na versão utilizada da Escala de Estratégias de Aprendizagem, com 37 itens. Acrescenta-se que para essa análise não se trabalhou com a amostra total, sendo que cerca de 50\% dos estudantes foram incluídos nessa etapa do estudo. Optou-se por trabalhar com a $A N O V A$, tendo em vista que as notas de tais disciplinas eram expressas em conceitos como: Em processo $(4,2 \% ; n=34)$, Realiza $(34,6 \% ; n=282)$ e Realiza plenamente $(14,5 \% ; n=118)$.

Pela análise dos dados na disciplina de português verificouse a existência de diferença estatisticamente significativa entre os grupos $\left[F_{(2,431)}=37,973 ; p \leq 0,001\right]$. O teste post-hoc de Tukey apontou que a diferença no desempenho da Escala de Estratégias de Aprendizagem estava entre os alunos que apresentaram o conceito 'Realiza plenamente' $(M=83,6)$ e aqueles que apresentaram o conceito 'Realiza' $(M=76,3 ; p \leq 0,001)$ ou 'Em processo' $(M=71,1 ; p \leq 0,001)$. Também houve diferença entre os alunos que obtiveram o conceito 'Realiza' e aqueles que obtiveram o conceito 'Em processo' $(p=0,005)$. 
Tabela 1. Distribuição dos itens por fator e suas respectivas cargas fatoriais.

\begin{tabular}{|c|c|c|c|c|}
\hline \multirow{2}{*}{ Itens } & \multirow{2}{*}{ Tipos } & \multicolumn{3}{|c|}{ Cargas Fatoriais } \\
\hline & & 1 & 2 & 3 \\
\hline EA1 & Ensaio/Cognitiva & & 0,478 & \\
\hline EA2 & Organização/Cognitiva & & 0,453 & \\
\hline EA3 & Ausência & 0,450 & & \\
\hline EA4 & Ensaio/Cognitiva & & 0,377 & \\
\hline EA5 & Elaboração/Cognitiva & & 0,484 & \\
\hline EA6 & Agrupamento no fator não previsto & & 0,464 & \\
\hline EA7 & Agrupamento no fator não previsto & & 0,329 & \\
\hline EA8 & Monitoramento/Metacognitiva & & & 0,390 \\
\hline EA9 & Ausência & 0,544 & & \\
\hline EA10 & Ausência & 0,594 & & \\
\hline EA11 & Cognitiva/Elaboração & & 0,528 & \\
\hline EA12 & Ensaio/Cognitiva & & 0,579 & \\
\hline EA13 & Elaboração/Cognitiva & & 0,568 & \\
\hline EA14 & Agrupamento no fator não previsto & & 0,449 & \\
\hline EA15 & Ausência & 0,448 & & \\
\hline EA16 & Agrupamento no fator não previsto & & 0,453 & \\
\hline EA17 & Monitoramento/Metacognitiva & & & 0,652 \\
\hline EA18 & Ensaio/Cognitiva & & 0,508 & \\
\hline EA19 & Ausência & 0,662 & & \\
\hline EA20 & Elaboração/Cognitiva & & 0,481 & \\
\hline EA21 & Elaboração/Cognitiva & & 0,476 & \\
\hline EA22 & Regulação/Metacognitiva & & & 0,436 \\
\hline EA23 & Ausência & 0,435 & & \\
\hline EA24 & Agrupamento no fator não previsto & & 0,347 & \\
\hline EA25 & Ensaio/Cognitiva & & 0,581 & \\
\hline EA26 & Ausência & 0,369 & & \\
\hline EA27 & Monitoramento/Metacognitiva & & & 0,562 \\
\hline EA28 & Ausência & 0,494 & & \\
\hline EA29 & Ausência & 0,583 & & \\
\hline EA30 & Ausência & 0,596 & & \\
\hline EA31 & Ausência & 0,646 & & \\
\hline EA32 & Regulação/Metacognitiva & & & 0,421 \\
\hline EA33 & Ausência & 0,561 & & \\
\hline EA34 & Agrupamento no fator não previsto & & 0,485 & \\
\hline EA35 & Monitoramento/ Metacognitiva & & & 0,698 \\
\hline EA36 & Ausência & 0,476 & & \\
\hline EA37 & Regulação/Metacognitiva & & & 0,489 \\
\hline
\end{tabular}

Tabela 2. Itens que deverão ser excluídos e respectivos tipos esperados no agrupamento.

\begin{tabular}{|c|c|c|}
\hline Item & Questão & Tipo \\
\hline 6 & $\begin{array}{l}\text { Depois que você estuda, costuma pedir } \\
\text { para alguém da sua casa lhe fazer } \\
\text { perguntas para ver se você aprendeu } \\
\text { mesmo? }\end{array}$ & $\begin{array}{l}\text { Regulação/ } \\
\text { metacognitiva }\end{array}$ \\
\hline 7 & $\begin{array}{l}\text { Quando você estuda consegue perceber } \\
\text { que não está aprendendo? }\end{array}$ & $\begin{array}{l}\text { Percepção/ } \\
\text { metacognitiva }\end{array}$ \\
\hline 14 & $\begin{array}{l}\text { Quando você tem muitas coisas da } \\
\text { escola para fazer num dia, costuma } \\
\text { fazer uma lista para não se esquecer de } \\
\text { nada ou para ver o que é melhor fazer } \\
\text { primeiro? }\end{array}$ & $\begin{array}{l}\text { Planejamento/ } \\
\text { metacognitiva }\end{array}$ \\
\hline 16 & $\begin{array}{l}\text { Quando você recebe uma nota de uma } \\
\text { prova, tenta fazer de novo as questões } \\
\text { que errou? }\end{array}$ & $\begin{array}{l}\text { Regulação/ } \\
\text { metacognitiva }\end{array}$ \\
\hline 24 & $\begin{array}{l}\text { Quando você está fazendo uma prova, } \\
\text { costuma ler suas respostas novamente } \\
\text { antes de entregar para o professor? }\end{array}$ & $\begin{array}{l}\text { Regulação/ } \\
\text { metacognitiva }\end{array}$ \\
\hline 34 & $\begin{array}{l}\text { Quando você está fazendo uma tarefa } \\
\text { difícil, costuma dizer para você mesmo: } \\
\text { 'Calma, eu vou conseguir'? }\end{array}$ & $\begin{array}{l}\text { Regulação/ } \\
\text { metacognitiva }\end{array}$ \\
\hline
\end{tabular}

Quanto às notas da disciplina matemática, $8,2 \%(n=67)$ dos alunos apresentaram o conceito 'Em processo', 35,8\% $(n=292)$ apresentaram o conceito 'Realiza' e 9,2\% $(n=75)$, o conceito 'Realiza plenamente'. Novamente, a ANOVA indicou diferença estatisticamente significativa entre os grupos $\left[F_{(2,431)}=21,772 ; p \leq 0,001\right]$. O teste post-hoc de Tukey indicou que a diferença no desempenho da Escala de Estratégias de Aprendizagem estava entre os alunos que apresentaram o conceito 'Realiza plenamente' $(M=82,1)$ e aqueles que apresentaram o conceito 'Realiza' $(M=78,1 ; p=0,003)$ ou 'Em processo' $(M=71,9 ; p \leq 0,001)$. Também houve diferença entre os alunos que obtiveram o conceito 'Realiza' e aqueles que apresentaram o conceito 'Em processo' $(p \leq 0,001)$.

\section{Discussão}

Os resultados demonstraram que, de um modo geral, os estudantes relataram recorrer a estratégias de aprendizagem no momento de estudo já que a média de pontos na escala foi de 79,3. Sobre esse aspecto, Boruchovitch (1999), Mckeachie e cols. (1985) e Reay (2006) enfatizam que o uso das estratégias de aprendizagem facilita a aprendizagem, uma vez que viabiliza a aquisição e a posterior recuperação e uso da informação, bem como fomenta o bom desempenho acadêmico.

Em relação à validade fatorial da Escala de Estratégias de Aprendizagem, de Boruchovitch e Santos (2004b), tal propriedade psicométrica pode ser aferida. A análise fatorial exploratória confirmou a estrutura de três fatores, conforme já apontado em estudos anteriores (Boruchovitch \& Santos, 2004a; Boruchovitch \& Santos, 2006; Boruchovitch \& cols. 
2006; Oliveira \& cols., 2006), embora a variância total explicada tenha sido baixa (29,4\%). Os alphas de Cronbach da escala toda $(0,83)$ e das subescalas Ausência de Estratégias de Aprendizagem $(0,79)$, Estratégias Cognitivas $(0,80)$ e Estratégias Metacognitivas $(0,62)$ foram considerados aceitáveis.

Sob esse aspecto, os itens 6, 7, 14, 16, 24 e 34 merecem atenção especial uma vez que carregaram em fator não previsto teoricamente. Esses itens apresentaram características associadas ao Fator Estratégias Metacognitivas, contudo se agruparam no Fator Estratégias Cognitivas. A maior parte dos itens era representativa de estratégias metacognitivas de regulação e dois itens se referiam a estratégias metacognitivas de planejamento e auto-percepção da aprendizagem. Presume-se que tais itens apresentavam problemas em sua formulação, o que certamente pode ter contribuído para uma confusão no entendimento de seu sentido por parte dos alunos. Cabe salientar que o Fator Estratégias Metacognitivas foi aquele que apresentou uma consistência interna menor $(0,62)$, embora aceitável, conforme observam Prieto \& Muñiz (2000). Além do problema mencionado referente à redação dos itens, outra hipótese possível refere-se à dificuldade ainda existente em se medir de forma mais acurada esse construto tão complexo, sobretudo em faixas etárias mais jovens, como a da amostra do presente estudo, a qual foi composta por alunos do Ensino Fundamental (Veenman \& cols. 2004).

Pondera-se, contudo, que outros itens da escala também se referiam a construtos correspondentes aos itens excluídos (portanto, não houve prejuízo), mas ao serem formulados de forma diferente, agruparam-se no fator esperado teoricamente. Assim, com os resultados obtidos no Fator Estratégias Metacognitivas, ainda que sejam excluídos os itens apresentados na Tabela 2, identifica-se que o alpha desse fator foi mais baixo que o dos demais. Destarte, ressalta-se a necessidade dos itens que compõem esse fator passarem por uma análise pormenorizada de seu conteúdo em estudos futuros.

No que se refere à evidência de validade concorrente da Escala de Estratégias de Aprendizagem, de Boruchovitch e Santos (2004b), obtida por meio de critério externo, a análise de variância indicou que tanto para a disciplina de português quanto para a de matemática, os estudantes que obtiveram o conceito 'Realiza plenamente' nas referidas disciplinas demonstraram uma melhor pontuação na escala. O mesmo foi percebido entre aqueles que obtiveram o conceito 'Realiza' em relação aos que obtiveram o conceito 'Em processo'. Enfatiza-se o fato de que tais disciplinas apresentavam conteúdos bastante diferentes que exigiam distintas habilidades e, ainda assim, em ambas, os alunos que mais pontuaram na escala foram aqueles que tinham um melhor desempenho acadêmico.

As evidências obtidas na presente pesquisa corroboram os dados obtidos por Loranger (1994), Moura (1992), OnatsuArvilommi e cols., (2002) e Purdie e Hattie (1996), uma vez que apontam que o bom desempenho acadêmico dos estudantes se relaciona com o uso de estratégias de aprendizagem no momento de estudo. Lamenta-se, contudo, que apesar de se verificar que o uso de estratégias de aprendizagem favorece o bom desempenho acadêmico, há uma carência de estudos de âmbito nacional que visem a construir e validar escalas de estratégias de aprendizagem. Assim sendo, destaca-se a necessidade de que novos estudos sejam realizados nos diversos segmentos da escolarização formal, a saber, Ensino Fundamental, Médio e Superior. Ao lado disso, é urgente que os resultados sejam amplamente divulgados, de modo a possibilitar o seu emprego em escala mais ampla, nas várias etapas de escolarização.

Ressalta-se que a presente pesquisa propôs o estudo de parâmetros psicométricos (índices de precisão e evidências de validade - fatorial e de critério) de uma escala de estratégias de aprendizagem destinada a alunos do Ensino Fundamental. Ao que parece, a escala aqui estudada apresenta ainda algumas imperfeições (nível de precisão de uma das subescalas) que devem ser mais bem investigadas de forma a subsidiar uma versão da escala ainda mais refinada e apropriada.

\section{Considerações Finais}

Para Almeida (2002), a aprendizagem é um processo ativo que deve ser fomentado por meio de métodos que permitam ao aluno o uso da iniciativa, primando, inclusive, pelo senso de responsabilidade do aluno. Desse modo, o uso de estratégias de aprendizagem, além de instrumentalizar o aluno para o momento do estudo, também permite, por meio das estratégias metacognitivas, que este planeje, monitore e regule o seu estudo para que o aprendizado seja potencializado.

Uma avaliação diagnóstica do uso de estratégias de aprendizagem poderia viabilizar programas interventivos que focariam o seu emprego e a sua utilização diversificada. Assim, essas propostas também deveriam atingir os professores, uma vez que serão estes os profissionais responsáveis por ensinar, em sala de aula, o uso de estratégias de aprendizagem.

Ao se discutir as implicações positivas do uso desse tipo de escala, destaca-se que no âmbito educacional, bem como em outros contextos, como o organizacional e o do trabalho, por exemplo, há muitas possibilidades de investigação sobre medidas de avaliação das estratégias de aprendizagem utilizadas. Entretanto, os estudos sobre novos instrumentos devem ser pensados à luz de um levantamento prévio que identifique o conhecimento acumulado até o momento, para que novas pesquisas com amostras mais amplas e diversificadas e em novos contextos sejam empreendidas.

\section{Referências}

Almeida, L. S. (2002). Facilitar a aprendizagem: ajudar os alunos a aprender e a pensar. Psicologia Escolar e Educacional, $6,155-165$.

Boruchovitch, E. (1999). Estratégias de aprendizagem e desempenho escolar: considerações para a prática educacional. Psicologia: Reflexão e Crítica, 12, 361-376.

Boruchovitch, E., \& Santos, A. A. A. (2004a). Escala de avaliação de estratégias de aprendizagem para crianças do ensino fundamental. Manuscrito não publicado, Universidade São Francisco, Bragança Paulista.

Boruchovitch, E., \& Santos, A. A. A. (2004b). Escala de avaliação de estratégias de aprendizagem para crianças do ensino fundamental. Manuscrito não publicado, Universidade São Francisco, Bragança Paulista. 
Boruchovitch, E., \& Santos, A. A. A. (2006). Estratégias de aprendizagem: conceituação e avaliação. Em A. P. P. Noronha \& F. F. Sisto (Orgs.), Facetas do fazer em avaliação psicológica (pp. 107-124). São Paulo: Vetor.

Boruchovitch, E., Santos, A. A. A., Costa, E. R., Neves, E. R. C., Cruvinel, M. Primi, R., \& Guimarães, S. E. R. (2006). Estudo preliminar para construção de uma escala de estratégias de aprendizagem infantil. Psicologia: Teoria e Pesquisa, 22, 297304.

Buriasco, R. L. C. (2000). Algumas considerações sobre avaliação educacional. Estudos em Avaliação Educacional, 22, 155-177.

Bzuneck, J. A. (2001). O esforço nas aprendizagens escolares: mais do que um problema motivacional do aluno. Revista Educação e Ensino - USF, 6, 7-18.

Bzuneck, J. A. (2004). Aprendizagem por processamento da informação: uma visão construtivista. Em E. Boruchovitch \& J. A. Bzuneck (Orgs.), Aprendizagem: processos psicológicos e contextos social na escola (pp. 17-54). Petrópolis: Vozes.

Costa, E. R., \& Boruchovitch, E. (2000). Fatores que influenciam o uso de estratégias de aprendizagem. Psico-USF, 5, 11-24.

Darsie, M. M. P. (1996). Avaliação e aprendizagem. Cadernos de Pesquisa, 99, 47-59.

Dembo, M. H. (1994). Applying educational psychology (5 ed.). New York: Longman.

Esteban, M. T. (2000). Exigências democráticas/exigências pedagógicas: avaliação. Tecnologia Educacional, 29, 3-6.

Jamieson, J. (1995). The cognitive styles of reflection/inpulsivity and field independence/dependence and ESL success. Em Brown, H. D. \& Gonzo, S. T. (Orgs.), Readings on second language acquisition (pp. 119-137). Illinois: Prentice Hall.

Kasai, R. C. B. (2000). Avaliação da aprendizagem: um projeto vivido. Revista Diálogo Educacional, 1, 41-49.

Loranger, A. L. (1994). The study strategies of successful and unsuccessful high school students. Journal of Reading Behavior, 26, 347-360.

Martins, R. C. (1999). Avaliação crítica de uma experiência de ensino aprendizagem. Estudos de Psicologia-PUC-Campinas, 16, 54-64.

Mckeachie, W. J., Pintrich, P. R., \& Lin, Y. G. (1985). Teaching learning strategies. Educational Psychologist, 20, 153-160.

Moura, E. V. X. (1992). Influência da abordagem e nível de proficiência no uso de estratégias por alunos bem e mal sucedidos. Dissertação de Mestrado, Universidade Estadual Paulista, Assis.

Nori, R., \& Giusberti, F. (2006). Predicting cognitive styles from spatial abilities. American Journal of Psychology, 119, 67-86.

Noronha, A. P. P. (1999). Avaliação psicológica: usos e problemas com ênfase nos testes. Tese de Doutorado, Universidade Católica de Campinas, Campinas.
Noronha, A. P. P., \& Alchieri, J. C. (2001). Reflexões sobre os instrumentos de avaliação psicológica. Em R. Primi (Org.), Temas em avaliação psicológica (pp. 7-16). Campinas: IBAP.

Oliveira, K. L., Boruchovitch, E., \& Santos, A. A. A. (2006) Escala de Estratégias de Aprendizagem: estudo das propriedades psicométricas. Em C. Machado, L. Almeida, M. A. Guisande, M. Gonçalves \& V. Ramalho (Orgs.), XI Conferência Internacional Avaliação Psicológica: formas e contextos (pp. 509-516). Braga: Psiquilibrios Edições.

Onatsu-Arvilommi, T., Nurmi, J. E., \& Aunola, K. (2002). The development of achievement strategies and academic skills during the first year of primary school. Learning an Instruction, 12, 509-527.

Prieto, G., \& Muñiz, J. (2000). Um modelo para evaluar la calidad de los tests utilizados em España. Retirado em 14/01/2006, de http://www.cop.es/tests/modelo.htm.

Purdie, N., \& Harttie, J. (1996). Cultural differences in the use of strategy for self-regulated learning. American Educational Reserarch Journal, 33, 845-871.

Reay, D. (2006). I'm not seen as one of the clever children: Consulting primary school pupils about the social conditions of learning. Educational Review, 58, 171-181.

Sisto, F. F., Sbardelini, E. T. B., \& Primi, R. (2001). Contextos e questões da avaliação psicológica. São Paulo: Casa do Psicólogo.

Sternberg, R. J. (2000). Psicologia cognitiva (M. R. B. Osório, Trad.). Porto Alegre: Artes Médicas. (Trabalho original publicado em 1996)

Tapia, J. A., \& Garcia-Celay, I. M. (1966). Motivação e aprendizagem escolar. Em C. Coll, J. Palácios \& A. Marchesi (Orgs.), Desenvolvimento psicológico e educação: psicologia da educação (pp. 161-175). Porto Alegre: Artes Médicas.

Valdés, M. T. M. (2003). Estrategias de aprendizaje: bases para la intervención psicopedagógica. Revista de Psicopedagogía, 20, 136-142.

Veenman, M. V. J., Wilhelm, P., \& Beishuizen, J. J. (2004). The relation between intellectual and metacognitive skills from a developmental perspective. Learning and Instruction, 14, 89-109.

Zimmerman, B. J., \& Martinez-Pons, M. (1986). Development of a structured interview for assessing student use of self-regulated learning strategies. American Educational Research Journal, 23, 614-628. 